\title{
ON WEYL'S TYPE THEOREMS AND GENERICITY OF PROJECTIVE RIGIDITY IN SUB-RIEMANNIAN GEOMETRY
}

\author{
FRÉDÉRIC JEAN, SOFYA MASLOVSKAYA, AND IGOR ZELENKO
}

\begin{abstract}
H. Weyl in 1921 ([8]) demonstrated that for a connected manifold of dimension greater than 1, if two Riemannian metrics are conformal and have the same geodesics up to a reparametrization, then one metric is a constant scaling of the other one. In the present paper we investigate the analogous property for sub-Riemannian metrics. In particular, we prove that the analogous statement, called the Weyl projective rigidity, holds either in real analytic category for all sub-Riemannian metrics on distributions with a specific property of their complex abnormal extremals, called minimal order, or in smooth category for all distributions such that all complex abnormal extremals of their nilpotent approximations are of minimal order. This also shows, in real analytic category, the genericity of distributions for which all sub-Riemannian metrics are Weyl projectively rigid and genericity of Weyl projectively rigid sub-Riemannian metrics on a given bracket generating distributions. Finally, this allows us to get analogous genericity results for projective rigidity of subRiemannian metrics, i.e. when the only sub-Riemannian metric having the same sub-Riemannian geodesics, up to a reparametrization, with a given one, is a constant scaling of this given one. This is the improvement of our results on the genericity of weaker rigidity properties proved in recent paper [5].
\end{abstract}

\section{Statement of the Problem and main Results}

In Riemannian geometry, projectively (or geodesically) equivalent metrics are Riemannian metrics on the same manifold which have the same geodesics, up to reparameterization. The local classification of all pairs of projectively equivalent Riemannian metrics under natural regularity assumptions was maid by Levi-Civita in 1896 ([7]). This paper is devoted to the projective equivalence of more general class of metrics, the sub-Riemannian metrics and is a continuation of our recent work [5].

A sub-Riemannian manifold is a triple $(M, D, g)$, where $M$ is a smooth connected manifold, $D$ is a distribution on $M$ (i.e. a subbundle of $T M$ ) which is assumed to be bracket generating everywhere in the sequel without special mentioning, and $g$ is a Riemannian metric on $D$, and thus defines an Euclidean structure on every fiber of $D$. We say that $g$ is a sub-Riemannian metric on $(M, D)$. Consider the optimal control problem of minimizing the corresponding energy functional $E(\gamma)=$ $\int g(\dot{\gamma}, \dot{\gamma}) d t$ on the space of absolutely continuous curve tangent to $D$. The geodesics of the sub-Riemannian metric $g$ are projections of the Pontryagin extremals for this

Date: January 24, 2020

2010 Mathematics Subject Classification. 53C17, 53A20, 53A30.

Key words and phrases. Sub-Riemannian geometry, Riemannian geometry, Conformal geometry, Projective Geometry, Normal Geodesics, Abnormal Geodesics, Nilpotent Approximation.

This work was supported by a public grant as part of the Investissement d'avenir project, reference ANR-11-LABX-0056-LMH, LabEx LMH, in a joint call with Programme Gaspard Monge en Optimisation et Recherche Opérationnelle, by the iCODE Institute project funded by the IDEX Paris-Saclay, ANR-11-IDEX-0003-02 and by the Grant ANR-15-CE40-0018 of the ANR. I. Zelenko was partly supported by NSF grant DMS-1406193 and Simons Foundation Collaboration Grant for Mathematicians 524213. 
problem. Sub-Riemannian Pontryagin extremals and the corresponding geodesics can be of two types, normal or abnormal.

The normal Pontryagin extremal of the sub-Riemannian metric are integral curves of the Hamiltonian system for the corresponding Hamiltonian $h$, living on a nonzero level set of this Hamiltonian. The Hamiltonian $h: T^{*} M \rightarrow \mathbb{R}$ of the sub-Riemannian metric $g$ is defined by

$$
h(q, p)=\frac{1}{2}\|p\|_{q}^{2}, \quad q \in M, p \in T_{q}^{*} M,
$$

where

$$
\|p\|_{q}=\max \{\langle p, v\rangle: v \in D(q), g(q)(v, v)=1\}, \quad p \in T_{q}^{*} M
$$

The abnormal Pontryagin extremals live in the zero level set of $h$, or, equivalent, on the annihilator $D^{\perp}$ of the distribution $D$, i.e.

$$
D^{\perp}=\left\{(q, p) \in T^{*} M: q \in M, p \in T_{q}^{*} M,\left.p\right|_{D(q)}=0\right\} .
$$

Their description is more involved, as they are not the integral curve of the subRiemannian Hamiltonian $h$, and will be given in section 5. Abnormal geodesics depend only on the distribution $D$, not on $g$, so they are automatically the same for all sub-Riemannian metrics on the same distribution.

Riemannian metrics appear as the particular case of sub-Riemannian ones, where $D=T M$. The classical Riemannian geodesics can be equivalently described as the normal geodesics coming from the corresponding Hamiltonian (1.1). Riemannian metrics do not have abnormal geodesics. We thus extend the definition of projectively equivalence to sub-Riemannian metrics in the following way.

Definition 1.1. Let $M$ be a manifold and $D$ be a bracket generating distribution on $M$. Two sub-Riemannian metrics $g_{1}$ and $g_{2}$ on $(M, D)$ are called projectively equivalent at $q_{0} \in M$ if they have the same geodesics, up to a reparameterization, in a neighborhood of $q_{0}$.

The trivial example of projectively equivalent metrics is the one of two constantly proportional metrics $g$ and $c g$, where $c>0$ is a real number. We thus say that these metrics are trivially (projectively or affinely) equivalent.

Definition 1.2. A sub-Riemannian metric $g$ on $(M, D)$ is said to be projectively rigid if it admits no non-trivially projectively equivalent metric.

It is still a widely open problem to classify all pairs of projectively equivalent sub-Riemannian metrics. A much easier task is to study whether the projectively rigid sub-Riemannian metrics form a generic set in the space of all sub-Riemannian metrics on a connected manifolds. In studying this question one naturally arrives to the following weaker (intermediate) notion of rigidity.

Definition 1.3. A sub-Riemannian metric $g$ is said to be conformally projectively rigid if any metric projectively equivalent to $g$ is conformal to $g$.

In our recent paper [5] we proved the following genericity results for conformally projective rigidity:

Theorem 1.4. Let $M$ be a smooth manifold and $D$ be a smooth distribution on $M$. A generic sub-Riemannian metric on $(M, D)$ is conformally projectively rigid.

Theorem 1.5. Let $m$ and $n$ be two integers such that $2 \leq m<n$, and assume $(m, n) \neq(4,6)$ and $m \neq n-1$ if $n$ is even. Then, given a smooth $n$ dimensional manifold $M$ and a generic smooth rank-m distribution $D$ on $M$, any sub-Riemannian metric on $(M, D)$ is conformally projectively rigid.

The latter theorem is based on the following result also proved in [5]. 
Theorem 1.6. If $D$ is a bracket generating distribution on a connected manifold $M$ such that the nilpotent approximation of it at every point of an open and dense subset of $M$ does not admit a product structure, then any sub-Riemannian metric on $D$ is conformally projectively rigid.

In light of these results, it is natural to ask whether conformally projective rigidity can be replaced by just projective rigidity in both of these theorems. In the Riemannian case H. Weyl in 1921 ([8]) demonstrated that for $\operatorname{dim} M>1$, if two Riemannian metrics are conformal and have the same geodesics up to a reparametrization, then one metric is a constant scaling of the other one. ${ }^{1}$

Definition 1.7. A metric $g$ is said to be Weyl projectively rigid if any metric, which is simultaneously conformal to $g$ and projectively equivalent to $g$ is constantly proportional to $g$.

So, in this terminology the Weyl theorem says that for $\operatorname{dim} M>1$ any Riemannian metric is Weyl projectively rigid. While in Riemannian case the proof of this result is rather trivial, it is not known yet whether the same statement is true for all sub-Riemannian metrics. The main problem that arises in trying to prove this statement for the general sub-Riemannian case is the presence of abnormal extremals. The main objective of the present paper is to study under what conditions are sub-Riemannian metrics Weyl projectively rigid. Studying the solvability of the equations for projective equivalence, one inevitably arrives to the questions of divisibility of certain polynomials on the fibers of the cotangent bundle $T^{*} M$, so it is natural to complexify the picture by complexifying not only the fibers of $T^{*} M$ but the manifold $M$ itself which is possible, at least locally, under assumption that $M$ is real analytic. This leads naturally to the necessity to consider the notion of complex extremals and geodesics. Very roughly speaking, we show that a real analytic sub-Riemannian metric is Weyl projectively rigid either if the underlying distribution does not have too much complex abnormal geodesics through a point or does not have too much complex non-strictly normal geodesics, i.e. complex normal geodesics which are simultaneously abnormal. Our condition are already enough to prove the Weyl projective rigidity for appropriate generic class of subRiemannian metrics in real analytic category (see Theorems 1.12 and 1.18) below) so that it is possible to replace conformally projective rigidity by projectively rigid in Theorems 1.4 and 1.5.

Now we will describe our results in more detail. Assume that $(M, D, g)$ is a real analytic sub-Riemannian structure. Locally (i.e. in a neighborhood of any points of $M$ ) we can consider a complex manifold $\mathbb{C} M$, a complexification of $M$, by extending the transition maps between charts, which are real analytic by definition, to analytic functions. We can extend locally the (real-analytic) distribution $D$ and sub-Riemannian metric $g$ to the (complex) analytic distribution $\mathbb{C} D$ and a field of symmetric forms $g^{\mathbb{C}}$ on each fiber of this distribution.

We can also consider the (complex) cotangent bundle $T^{*} \mathbb{C} M$ of $\mathbb{C} M$ whose fibers are complex vector spaces. We can extend the sub-Riemannian Hamiltonian $h$ defined by (1.1) analytically to the complex Hamiltonian $h^{\mathbb{C}}$ on this bundle and consider the corresponding complex Hamiltonian vector field. The complex normal extremals are by definition the integral curves of this vector field and the complex normal geodesics are projections of these integral curves to $\mathbb{C} M$.

Remark 1.8. Note that after the complexification the zero-level set $\left(h^{\mathbb{C}}\right)^{-1}(0)$ of the complex sub-Riemannian Hamiltonian $h^{\mathbb{C}}$ is strictly larger that the annihilator

\footnotetext{
${ }^{1}$ In fact this is a simple consequence of the Levi-Civita classification in [7] which was written much earlier than [8] but we prefer to relate it first to $\mathrm{H}$. Weyl as the great founder of both conformal and projective geometry.
} 
$\mathbb{C} D^{\perp}$. The integral curves of the complexified Hamiltonian lying in $\left(h^{\mathbb{C}}\right)^{-1}(0) \backslash \mathbb{C} D^{\perp}$ play the same role as null geodesics in the pseudo-Riemannian geometry and, in particular, they are the same, up to reparameterization, for all sub-Riemannian metrics from the same conformal class. We will call them and their projections to $\mathbb{C} M$ the complex null normal extremals and geodesics, respectively.

Also, we can define Jacobi curve and the corresponding osculating flag for every complex normal extremal. Further, manipulating with the annihilators $(\mathbb{C} D)^{\perp}$ of the complex distribution $\mathbb{C} D$ in the complex cotangent bundle of $\mathbb{C} M$, similarly to the standard real case, we can define complex abnormal, and consequently the strictly normal sub-Riemannian geodesics (see section 5 for more detail).

We also need the notion of a corank of a geodesics. From now on by dimensions we will mean complex dimensions. Given a complex normal geodesic $\gamma$ of a subRiemannian metric $g$ we say that a complex Pontryagin normal extremal projected to $\gamma$ as parameterized curve is a Pontryagin normal lift of $\gamma$. Given an abnormal geodesic $\gamma$ of a distribution $D$ we say that a complex Pontryagin abnormal extremal projected to $\gamma$ is a Pontryagin abnormal lift of $\gamma$. The projection of this lift to the projectivized cotangent bundle $\mathbb{P} T^{*} M$ will be called the projectivized Pontryagin abnormal lift of $\gamma$. The corank of a complex normal geodesics $\gamma$ of a sub-Riemannian metric $g$ is by definition the dimension of the affine space of the normal Pontryagin lifts of $\gamma$. The corank of a normal geodesic is a nonnegative integer.

The corank of a complex abnormal geodesic $\gamma$ of a distribution $D$ is by definition the dimension of the (vector) space of all its abnormal lifts. The corank of an abnormal geodesic is a positive integer.

Remark 1.9. Note that if $\left(\gamma(t), p_{1}(t)\right)$ and $\left(\gamma(t), p_{2}(t)\right)$ are two distinct normal lifts of a normal geodesics $\gamma$ (which are either both null or both non-null), then $\left(\gamma(t), p_{2}(t)-p_{1}(t)\right)$ is an abnormal lift of $\gamma$. Similarly, if $\left(\gamma(t), p_{1}(t)\right)$ is an abnormal lift of a geodesic $\gamma$ and $\left(\gamma(t), p_{2}(t)\right)$ is a normal lift of $\gamma$, then $\left(\gamma(t), p_{1}(t)+p_{2}(t)\right)$ is a normal lift of $\gamma$.

From the previous remark it follows that a normal geodesic $\gamma$ is simultaneously an abnormal geodesic if and only if its corank is greater than 0 and in this case the corank of $\gamma$ as normal geodesics is equal to the corank of $\gamma$ as abnormal geodesics.

Further, if given positive integers $\kappa$ and $s$, there exists a nonzero number $c \in$ $\mathbb{C}$ and a $(s+\kappa+1)$-dimensional submanifold of the $c$-level set $\left(h^{\mathbb{C}}\right)^{-1}(c)$ of the complexified sub-Riemannian Hamiltonian $h^{\mathbb{C}}$ which is foliated by complex normal extremals of corank $\kappa$, then we say that the projections of these extremals to $M$ form an s-parametric family of complex normal geodesics of corank $\kappa$.

Also note that the nilpotent approximation (even of a smooth but not real analytic sub-Riemannian structure) is always real analytic as it is a left-invariant structure on a nilpotent Lie group. The following two theorems are our main results on Weyl projective rigidity in terms of normal geodesics:

Theorem 1.10. Assume that $(M, D, g)$ is a smooth sub-Riemannian manifold such that its nilpotent approximation at every point of an open and dense subset of $M$ satisfies the following property: for every positive $\kappa \in \mathbb{N}$, there is no $(n-2-\kappa)$ parametric family of corank $\kappa$ non-strictly normal complex geodesics through a point. Then the metric $g$ is Weyl projectively rigid.

Theorem 1.11. Assume that $(M, D, g)$ is a real analytic sub-Riemannian manifold such that there is no open set $U$ in $\mathbb{C} M$ with the following property: for some positive integer $\kappa \leq n-2$ through any point $q \in U$ there is an $(n-2-\kappa)$-parametric family of corank $\kappa$ non-strictly normal complex geodesics. Then the metric $g$ is Weyl projectively rigid. 
These two theorems will be proved in section 4, based on more general but technical Theorem 3.2, proved in section 3.

The conclusion of Theorems 1.10 and 1.11 for a sub-Riemannian structure holds in particular when either the sub-Riemannian structure is smooth, and its nilpotent approximation do not have complex non-strictly normal geodesics or the subRiemannian structure is real analytic and does not have complex non-strictly normal geodesics. The latter holds generically. It follows from the complex analog of [3, Proposition 2.22], which has literally the same proof. To summarize, we have the following result on genericity of the Weyl rigidity:

Corollary 1.12. Let $M$ be a real analytic manifold and $D$ be a real analytic distribution on $M$ of rank greater than 1. A generic real analytic sub-Riemannian metric on $(M, D)$ is Weyl rigid.

Now we formulate our main results on Weyl projective rigidity in terms of complex abnormal extremals. We will use the notion of an abnormal extremal of minimal order, introduced in [2], see Definition 5.2 below. The condition of minimal order implies in particular that on a set of full measure such abnormal extremal is tangent to a prescribed line or equivalently, on a set of full measure the germ of the extremal at any point of this set is uniquely determined by this point. The following two theorems will be proved in section 5 .

Theorem 1.13. Assume that $D$ is a smooth distribution on a connected manifold $M$ such that its nilpotent approximation at every point of an open and dense subset of $M$ satisfies the following properties: every complex abnormal extremal of the nilpotent approximation is of minimal order. Then any smooth sub-Riemannian metric on $D$ is Weyl projectively rigid.

Theorem 1.14. Assume that $D$ is a real analytic distribution on a connected manifold such that every complex abnormal extremal of $D$ is of minimal order. Then any real analytic sub-Riemannian metric on $D$ is Weyl projectively rigid.

The direct consequence of Theorems 1.6 and 1.13 is the following.

Corollary 1.15. If $D$ is a smooth bracket generating distribution on a connected manifold $M$ such that the nilpotent approximation $\hat{D}$ of it at every point of an open and dense subset of $M$ does not admit a product structure and every complex abnormal extremal of $\hat{D}$ is of minimal order, then any smooth sub-Riemannian metric on $D$ is projectively rigid.

Based on the last corollary we can easily find many new classes of distributions on connected manifolds for which all sub-Riemannian metrics on them are projectively rigid (before this statement was known for contact distributions only ([9])). For example, this will be true for the following distributions, for which it is easy to see that all possible nilpotent approximations satisfy conditions of Corollary 1.15:

(1) Engel distributions, i.e. rank 2 distributions on 4-dimensional manifolds with the small growth vector $(2,3,4)$;

(2) Rank 2 distributions on 5-dimensional manifolds with the small growth vectors $(2,3,5)$;

(3) rank 3 distributions on 5-dimensional and 6 dimensional manifolds with the small growth vectors $(3,5)$ and $(3,6)$, respectively;

(4) Rank 2 distributions on 6 dimensional manifolds with the small growth vectors $(2,3,5,6)$;

(5) Rank 2 distributions on 7 dimensional manifolds with the small growth vectors $(2,3,5,7)$ or $(2,3,5,6,7)$. 
Remark 1.16. Conditions of minimal order in Theorems 1.13 and 1.14 can be replaced by much weaker but much more technically formulated condition, see subsection 5.5.

Further, the main result of [2, Theorem 2.4] states that all (real) abnormal extremals of a generic smooth rank $m$ distribution are of minimal order and corank 1. This result can be literally extended to the complex abnormal exttremals of real analytic manifolds), because the genericity condition in [2, Theorem 2.4] is given by the complement of algebraic conditions with respect to the fibers of $T^{*} M$ so that the complexification can be done. So, the following theorem holds.

Theorem 1.17. All complex abnormal extremals of a generic rank $m$ real analytic distribution distribution are of minimal order and corank 1.

Combining this theorem with Theorem 1.13 we get one more genericity results on the Weyl rigidity.

Corollary 1.18. Let $m$ and $n$ be two integers such that $2 \leq m<n$. On a generic real analytic rank $m$ distribution $D$ on a connected $n$-dimensional real analytic manifold $M$ any sub-Riemannian metric is Weyl projectively rigid.

Finally, as immediate consequences of Theorems 1.4 and 1.12 and Theorems 1.5 and 1.18, respectively, we get the following two genericity results for projective rigidity, improving the main results of [5].

Corollary 1.19. Let $M$ be a real analytic manifold and $D$ be a distribution on $M$. A generic real analytic sub-Riemannian metric on $(M, D)$ is projectively rigid.

Corollary 1.20. Let $m$ and $n$ be two integers such that $2 \leq m<n$, and assume $(m, n) \neq(4,6)$ and $m \neq n-1$ if $n$ is even. Then, given a smooth $n$ dimensional manifold $M$ and a generic smooth rank $m$ distribution $D$ on $M$, any sub-Riemannian metric on $(M, D)$ is projectively rigid.

\section{The FUndamental Algebraic SYStem in The CONFORMAL CASE}

2.1. Equations for orbital diffeomorphisms in local coordinates. In this subsection, following [5], we introduce orbital diffeomorphisms between extremal flows and explain their relation to the projective equivalence, then deduce the equations for orbital diffeomorphisms in local metric for projective equivalent and conformal sub-Riemannian metric. All formulas here can be directly derived from the corresponding formulas in [9] and [5], where the general case (of not necessary conformal but projective equivalent sub-Riemannian metrics) is considered. To make the presentation self-contained we derive all formulas here in the particular conformal case.

Let $M$ be a manifold and $D$ be a bracket generating distribution on $M$. We consider two sub-Riemannian metrics on $(M, D)$ that are both conformal and projectively equivalent. Let us denote these metrics by $g$ and $\alpha^{2} g$, where $\alpha: M \rightarrow \mathbb{R}$ is a never vanishing smooth function. Let $h_{1}$ and $h_{2}$ be the sub-Riemannian Hamiltonians of $g$ and $\alpha^{2} g$, respectively. Obviously

$$
h_{2}=\frac{1}{\alpha^{2}} h_{1} .
$$

Denote $H_{1}=h_{1}^{-1}(1 / 2)$ and $H_{2}=h_{2}^{-1}(1 / 2)$ the respective $\frac{1}{2}$ level sets of these Hamiltonians. Also let $\pi: T^{*} M \rightarrow M$ be the canonical projection.

One says that $\vec{h}_{1}$ and $\vec{h}_{2}$ are orbitally diffeomorphic on an open subset $V_{1}$ of $H_{1}$ if there exists an open subset $V_{2}$ of $H_{2}$ and a diffeomorphism $\Phi: V_{1} \rightarrow V_{2}$ such that $\Phi$ is fiber-preserving, i.e. $\pi(\Phi(\lambda))=\pi(\lambda)$, and $\Phi$ sends the integral curves of 
$\vec{h}_{1}$ to the reparameterized integral curves of $\vec{h}_{2}$, i.e., there exists a smooth function $s=s(\lambda, t)$ with $s(\lambda, 0)=0$ such that $\Phi\left(e^{t \vec{h}_{1}} \lambda\right)=e^{s \vec{h}_{2}}(\Phi(\lambda))$ for all $\lambda \in V_{1}$ and $t \in \mathbb{R}$ for which $e^{t \vec{h}_{1}} \lambda$ is well defined. Equivalently, there exists a smooth function $c(\lambda)$ such that

$$
d \Phi \circ \vec{h}_{1}(\lambda)=c(\lambda) \vec{h}_{2}(\Phi(\lambda)) .
$$

The map $\Phi$ can be extended as a mapping $\bar{\Phi}$ from $T^{*} M \backslash h_{1}^{-1}(0)$ to itself by rescaling, i.e.,

$$
\bar{\Phi}(\lambda)=\sqrt{2 h_{1}(\lambda)} \Phi\left(\frac{\lambda}{\sqrt{2 h_{1}(\lambda)}}\right) .
$$

The resulting map is called an orbital diffeomorphism between the extremal flows of $g$ and $\alpha^{2} g$. In the considered case, from (2.1) and the fact that $\Phi$ is fiber-preserving it follows immediately that the function $c(\lambda)$ in $(2.2)$ coincides with the function $\alpha \circ \pi(\lambda))$, i.e. we have

$$
d \Phi \circ \vec{h}_{1}(\lambda)=\alpha \circ \pi(\lambda) \vec{h}_{2}(\Phi(\lambda)) .
$$

In [5] we established the relationship between projective equivalence of subRiemannian and orbital equivalence of the corresponding sub-Riemannian Hamiltonians. In particular, in Proposition 3.4 there we proved that there exists a local orbital diffeomorphisms $\Phi$ between the Hamiltonian vector fields associated with $g$ and $\alpha^{2} g$ near generic ${ }^{2}$ point of $T^{*} M$.

Now we will work in coordinates on fibers of $T^{*} M$ induced by an appropriate local moving frame on $M$. Fix a point $q_{0} \in M$ and choose a frame $\left\{X_{1}, \ldots, X_{n}\right\}$ of $T M$ adapted to $D$ at $q_{0}$ such that $X_{1}, \ldots, X_{m}$ is a $g$-orthonormal frame of $D$. At any point $q$ in a neighborhood $U$ of $q_{0}$, the basis $X_{1}(q), \ldots, X_{n}(q)$ of $T_{q} M$ induces coordinates $\left(u_{1}, \ldots, u_{n}\right)$ on $T_{q}^{*} M$ defined as $u_{i}(q, p)=\left\langle p, X_{i}(q)\right\rangle$. These coordinates in turn induce a basis $\partial_{u_{1}}, \ldots, \partial_{u_{n}}$ of $T_{\lambda}\left(T_{q}^{*} M\right)$ for any $\lambda \in \pi^{-1}(q)$. For $i=1, \ldots, n$, we define the lift $Y_{i}$ of $X_{i}$ as the (local) vector field on $T^{*} M$ such that $\pi_{*} Y_{i}=X_{i}$ and $d u_{j}\left(Y_{i}\right)=0 \quad \forall 1 \leq j \leq n$. In this way the local frame $\left\{X_{1}, \ldots, X_{n}\right\}$ on $M$ induces the local frame

$$
\left\{Y_{1}, \ldots, Y_{n}, \partial_{u_{1}}, \ldots, \partial_{u_{n}}\right\}
$$

on $T^{*} M$. By a standard calculation, we obtain $h_{1}=\frac{1}{2} \sum_{i=1}^{m} u_{i}^{2}$ and

$$
\vec{h}_{1}=\sum_{i=1}^{m} u_{i} Y_{i}+\sum_{i=1}^{m} \sum_{j, k=1}^{n} c_{i j}^{k} u_{i} u_{k} \partial_{u_{j}}
$$

where $c_{i j}^{k}, i, j, k \in\{1, \ldots, n\}$ are the structure functions of the frame $\left\{X_{1}, \ldots, X_{n}\right\}$, defined near $q_{0}$ by

$$
\left[X_{i}, X_{j}\right]=\sum_{k=1}^{n} c_{i j}^{k} X_{k}
$$

Further, from (2.1) it follows that $\vec{h}_{2}=\frac{1}{\alpha^{2}} \vec{h}_{1}+h_{1} \overrightarrow{\left(\frac{1}{\alpha^{2}}\right)}$ and so

$$
\vec{h}_{2}=\frac{1}{\alpha^{2}}\left(\sum_{i=1}^{m} u_{i} Y_{i}+\sum_{i=1}^{m} \sum_{j, k=1}^{n} c_{i j}^{k} u_{i} u_{k} \partial_{u_{j}}\right)-\sum_{j=1}^{n} \frac{1}{\alpha} X_{j}\left(\frac{1}{\alpha}\right)\left(\sum_{i=1}^{m} u_{i}^{2}\right) \partial_{u_{j}} .
$$

Finally, let us denote by $\Phi_{i}(\lambda), i=1, \ldots, n$, the $u_{i}$-component of $\Phi(\lambda)$ on the fiber, i.e. $\Phi_{i}(\lambda)=u_{i} \circ \Phi(\lambda)$. From (2.1) it follows that

$$
\Phi_{k}=\alpha u_{k} \quad \text { for } k=1, \ldots, m .
$$

\footnotetext{
${ }^{2}$ In fact in the original formulation in [5] we used the term ample instead of generic, see [Definition 2.9] there, but we do not really need this technicalities here.
} 
Substituting this into (2.3) we get the following: ${ }^{3}$

Lemma 2.1. The map $\Phi$ is an orbital diffeomorphism between extremal flows of sub-Riemannian metrics $g$ and $\alpha^{2} g$ if and only if the components $\Phi_{m+1}, \ldots, \Phi_{n}$ satisfy the following system of equations:

$$
\begin{aligned}
& \text { (2.8) } \sum_{k=m+1}^{n} q_{j k}\left(\Phi_{k}-\alpha u_{k}\right)=\sum_{i=1}^{m}\left(X_{i}(\alpha) u_{j}-X_{j}(\alpha) u_{i}\right) u_{i}, \quad j=1, \ldots, m \\
& (2.9) \quad \vec{h}_{1}\left(\Phi_{k}-\alpha u_{k}\right)=\sum_{l=m+1}^{n} q_{k l}\left(\Phi_{l}-\alpha u_{l}\right)+ \\
& \quad+\sum_{i=1}^{m}\left(X_{k}(\alpha) u_{i}-X_{i}(\alpha) u_{k}\right) u_{i}, \quad k=m+1, \ldots, n,
\end{aligned}
$$

where $q_{j k}=\sum_{i=1}^{m} c_{i j}^{k} u_{i}$.

Equations (2.8)-(2.9) are obtained by straightforward calculations in the moving frame (2.4) after plugging equations (2.5), (2.6), and (2.7) into (2.3). Equations (2.8) are obtained by comparison of the components of $\partial_{u_{j}}$ of both sides of (2.3) with $j=1, \ldots, m$, while equations $(2.9)$ are obtained by comparison of the components of $\partial_{u_{k}}$ of both sides of $(2.3)$ with $k=1, \ldots, m+1$.

2.2. Fundamental algebraic system. Now following [5] again we replace the system (2.8)-(2.9) that contains derivatives of the unknown functions $\Phi_{k}, k=$ $m+1, \ldots, n$ by the (infinite) linear algebraic i.e. without derivatives) system for that unknown function that we call the fundamental algebraic system. The process of obtaining the latter can be seen in a sense as the infinite prolongation of the subsystem given by (2.8) using in each step of the prolongation the equations from $(2.9)$.

In more details, in the first step one differentiate each of $m$ equations from (2.8) in the direction of $h_{1}$ and replace each $\vec{h}_{1}\left(\Phi_{k}-\alpha u_{k}\right)$ in the resulting expression by the right-hand side of (2.9). In this way we get new $m$ equations which are linear in $\Phi_{k}-\alpha u_{k}$. In the next step we differentiate these new $m$ equations in the direction of $h_{1}$ and replace each $\vec{h}_{1}\left(\Phi_{k}-\alpha u_{k}\right)$ in the resulting expression by the right-hand side of (2.9) to obtain new $m$ equations which are linear in $\Phi_{k}-\alpha u_{k}$. The fundamental algebraic system is obtained by repeating this process infinitely many times. Setting $\widetilde{u}=\left(u_{m+1}, \ldots, u_{n}\right)$ and $\widetilde{\Phi}=\left(\Phi_{m+1}, \ldots, \Phi_{n}\right)$, the fundamental algebraic system $[5,(3.8)]$ writes as

$$
A(\widetilde{\Phi}-\alpha \widetilde{u})=d
$$

where $A$ is the matrix defined recursively in [5, (3.10)] and $d$ is a column vector with an infinite number of rows which can be decomposed in layers of $m$ rows as

$$
d=\left(\begin{array}{c}
d^{1} \\
d^{2} \\
\vdots \\
d^{s} \\
\vdots
\end{array}\right)
$$

\footnotetext{
${ }^{3}$ From now on to simplify the notation in all relation involving functions on open sets of $T^{*} M$ $\alpha$ actually will mean $\alpha \circ \pi$.
} 
where the coefficients $d_{j}^{s}, 1 \leq j \leq m$, of the vector $d^{s} \in \mathbb{R}^{m}$ are defined by

$$
\left\{\begin{array}{l}
d_{j}^{1}=\sum_{i=1}^{m}\left(X_{i}(\alpha) u_{j}-X_{j}(\alpha) u_{i}\right) u_{i} \\
d_{j}^{s+1}=\vec{h}_{1}\left(d_{j}^{s}\right)+\sum_{k=m+1}^{n} a_{j, k}^{s} \sum_{i=1}^{m} u_{i}\left(X_{i}(\alpha) u_{k}-X_{k}(\alpha) u_{i}\right) .
\end{array}\right.
$$

Note that by [5, Proposition 3.11] the matrix $A(u)$ is injective at a generic $u$.

2.3. Sufficient conditions for Weyl rigidity in terms of solutions of the fundamental algebraic system. The fundamental algebraic system (2.10) implies that the coordinates of $\Phi$ are rational functions on the fibers. Proving that $g$ and $\alpha^{2} g$ are proportional actually amounts to prove that these coordinates are polynomial, as stated below.

Proposition 2.2. If there exists a local orbital diffeomorphism $\Phi$ which is polynomial on the fibers, then $g$ and $\alpha^{2} g$ are locally constantly proportional, i.e., $\alpha$ is constant.

Before giving the proof of this result, we need to study the consequence of the fundamental algebraic system on the nilpotent approximation.

Fix a regular point $q_{0}$ and denote by $(\hat{M}, \hat{D})$ the nilpotent approximation of $(M, D)$ at $q_{0}$. We argue as in the proof of [5, Theorem 7.1], with the same notations. In particular $\left\{\hat{X}_{1}, \ldots, \hat{X}_{n}\right\}$ is a frame of $T \hat{M}$ adapted to $\hat{D}$ such that $\hat{X}_{1}, \ldots, \hat{X}_{m}$ is $\hat{g}$-orthonormal and $\hat{A}$ is the matrix of [5, Proposition 3.10] constructed by using $\left\{\hat{X}_{1}, \ldots, \hat{X}_{n}\right\}$ as a frame.

Lemma 2.3. There exists one, and only one, solution $\Psi=\left(\Psi_{m+1}, \ldots, \Psi_{n}\right)$ s of

$$
\hat{A} \Psi=\hat{d}
$$

where, for any $s \in \mathbb{N}$ and $1 \leq j \leq m, \hat{d}_{j}^{s}$ is defined by

$$
\left\{\begin{array}{l}
\hat{d}_{j}^{1}=\sum_{i=1}^{m}\left(X_{i}(\alpha)\left(q_{0}\right) u_{j}-X_{j}(\alpha)\left(q_{0}\right) u_{i}\right) u_{i} \\
\hat{d}_{j}^{s+1}=\overrightarrow{\hat{h}}_{1}\left(\hat{d}_{j}^{s}\right)+\sum_{k=m+1}^{n} \hat{a}_{j, k}^{s} \sum_{i=1}^{m} u_{i} X_{i}(\alpha)\left(q_{0}\right) u_{k} .
\end{array}\right.
$$

Proof. An easy induction argument based on equations (2.11) shows the following result, similar to [5, Lemma 7.4]: for any $s \in \mathbb{N}$ and $1 \leq j \leq m$, there hold:

- for every $q \in M$ near $q_{0}, d_{j}^{s}$ is a polynomial in $u_{1}, \ldots, u_{n}$ of weighted degree

$$
\operatorname{deg}_{w}\left(d_{j}^{s}\right) \leq 2 s
$$

- the homogeneous term of highest weighted degree in $d_{j}^{s}\left(q_{0}\right)$ is $\hat{d}_{j}^{s}$.

It results from $(2.10)$ that $\left(\begin{array}{ll}A & d\end{array}\right)$ is not of full rank, thus also the matrix $\left(\begin{array}{ll}\hat{A} & \hat{d}\end{array}\right)$ is not of full rank. Since $\hat{A}$ is of full rank at a generic $u$ by [5, Proposition 3.11], there exists a unique element in $\operatorname{ker}\left(\begin{array}{ll}\hat{A} & \hat{d}\end{array}\right)$ of the form $(\Psi,-1)$, which ends the proof.

Using all equations above it is easy to show that $\Psi$ has the following properties.

(i) Each $\Psi_{k}, k=m+1, \ldots, n$, is a rational function which is:

- homogeneous of degree 1 w.r.t. the usual degree;

- $w$-homogeneous with $\operatorname{deg}_{w}\left(\Psi_{k}\right)=w_{k}-1$. 
(ii) For $j=1, \ldots, m$, we have

$$
\sum_{\left\{k: w_{k}=2\right\}} \sum_{i=1}^{m} \hat{c}_{i j}^{k} u_{i} \Psi_{k}=\sum_{i=1}^{m}\left(\alpha^{i} u_{j}-\alpha^{j} u_{i}\right) u_{i}
$$

where $\alpha^{j}=X_{i}(\alpha)\left(q_{0}\right)$.

(iii) For $k=m+1, \ldots, n$, we have

$$
\overrightarrow{\hat{h}}\left(\Psi_{k}\right)=\sum_{\left\{l: w_{l}=w_{k}+1\right\}} \sum_{i=1}^{m} \hat{c}_{i k}^{l} u_{i} \Psi_{l}-\sum_{i=1}^{m} \alpha^{i} u_{k} u_{i} .
$$

Lemma 2.4. Assume that the map $\Psi$ given in Lemma 2.3 is polynomial. Then

$$
X_{1}(\alpha)\left(q_{0}\right)=\cdots=X_{m}(\alpha)\left(q_{0}\right)=0 .
$$

Proof. By hypothesis, every $\Psi_{k}, k=m+1, \ldots, n$, is a polynomial. Moreover, by Property (i) above, $\Psi_{k}$ is a linear function of $u$ and depends only on the coordinates $u_{l}$ of weight $w_{l}=w_{k}-1$. To simplify the notations, we use the following convention: given a positive integer $s$, an index $k_{s}$ denotes an index of weight $w_{k_{s}}=s$ and $\sum_{k_{s}}$ denotes $\sum_{\left\{k_{s}: w_{k_{s}}=s\right\}}$. With this notation we have, for every $k_{s}$,

$$
\Psi_{k_{s}}=\sum_{k_{s-1}} \varepsilon_{k_{s} k_{s-1}} u_{k_{s-1}}
$$

where the coefficients $\varepsilon_{k_{s} k_{s-1}}$ are real numbers. Taking the derivative along $\overrightarrow{\hat{h}}$ we obtain

$$
\overrightarrow{\hat{h}}\left(\Psi_{k_{s}}\right)=\sum_{i=1}^{m} \sum_{k_{s-1}, l_{s}} \varepsilon_{k_{s} k_{s-1}} \hat{c}_{i k_{s-1}}^{l_{s}} u_{i} u_{l_{s}} .
$$

On the other hand, plugging (2.14) into (2.13), we get

$$
\overrightarrow{\hat{h}}\left(\Psi_{k_{s}}\right)=\sum_{i=1}^{m} \sum_{l_{s}, l_{s+1}} \hat{c}_{i k_{s}}^{l_{s+1}} \varepsilon_{l_{s+1} l_{s}} u_{i} u_{l_{s}}-\sum_{i=1}^{m} \alpha^{i} u_{k} u_{i} .
$$

Fix an index $i \in\{1, \ldots, m\}$. By identifying the coefficients of the monomial $u_{i} u_{k_{s}}$ in (2.15) and (2.16), we obtain the following equality,

$$
\sum_{k_{s-1}} \varepsilon_{k_{s} k_{s-1}} \hat{c}_{i k_{s-1}}^{k_{s}}=\sum_{k_{s+1}} \hat{c}_{i k_{s}}^{k_{s+1}} \varepsilon_{k_{s+1} k_{s}}-\alpha^{i}
$$

and, after a summation on the $n_{s}-n_{s-1}$ indices $k_{s}$,

$$
\sum_{k_{s-1}, k_{s}} \hat{c}_{i k_{s-1}}^{k_{s}} \varepsilon_{k_{s} k_{s-1}}=\sum_{k_{s}, k_{s+1}} \hat{c}_{i k_{s}}^{k_{s+1}} \varepsilon_{k_{s+1} k_{s}}-\left(n_{s}-n_{s-1}\right) \alpha^{i} .
$$

Set $K_{i}(s)=\sum_{k_{s}, k_{s+1}} \hat{c}_{i k_{s}}^{k_{s+1}} \varepsilon_{k_{s+1} k_{s}}$. Then the above equality writes as

$$
K_{i}(s-1)=K_{i}(s)-\left(n_{s}-n_{s-1}\right) \alpha^{i} \quad \text { for } s>1 .
$$

Note that $K_{i}(r)=0$ since $r$ is the nilpotency step. Hence,

$$
K_{i}(1)=-\left(n_{r}-n_{1}\right) \alpha^{i}=-(n-m) \alpha^{i} .
$$

Now, by plugging (2.14) in (2.12), we have, for $j=1, \ldots, m$ :

$$
\sum_{k_{2}, k_{1}} \sum_{i=1}^{m} \hat{c}_{i j}^{k_{2}} \varepsilon_{k_{2} k_{1}} u_{i} u_{k_{1}}=\sum_{i=1}^{m}\left(\alpha^{i} u_{j}-\alpha^{j} u_{i}\right) u_{i}
$$


Given an index $i \in\{1, \ldots, m\}$, the identification of coefficient of $u_{i} u_{j}$ in this equality gives

$$
\sum_{k_{2}} \hat{c}_{i j}^{k_{2}} \varepsilon_{k_{2} j}=\left(1-\delta_{i j}\right) \alpha^{i}
$$

and by summation on the indices $j=k_{1}$, we obtain

$$
K_{i}(1)=(m-1) \alpha^{i} .
$$

This equation and (2.17) imply $\alpha^{i}=0$, which ends the proof.

Proof of Proposition 2.2. Assume $\Phi$ to be defined on an open subset $U$ of $T^{*} M$. Fix a regular point $q_{0}$ in $\pi(U)$ and let $(\hat{M}, \hat{D})$ be the nilpotent approximation of $(M, D)$ at $q_{0}$.

Let $\hat{\delta}$ be a nonzero maximal minor of $\hat{A}$. It is a $w$-homogeneous polynomial which is the homogeneous part of highest weighted degree of the corresponding minor (same rows and columns) $\delta$ of $A$, which is nonzero as well. It results easily from (2.10) that, for $k=m+1, \ldots, n$, we have $\Phi_{k}-\alpha u_{k}=p_{k} / \delta$ where $d_{w}\left(p_{k}\right) \leq d_{w}(\delta)+2$, and $\Psi_{k}=\hat{p}_{k} / \hat{\delta}$, where $\hat{p}_{k}$ is the homogeneous part (eventually zero) of weighted degree $d_{w}(\delta)+2$ in $p_{k}$. From the hypothesis of the theorem, $p_{k} / \delta$ is polynomial, therefore $\Psi_{k}=\hat{p}_{k} / \hat{\delta}$ is polynomial as well and by Lemma 2.4 we get $X_{i}(\alpha)\left(q_{0}\right)=0$, $i=1, \ldots, m$.

Since regular points form an open and dense subset of $\pi(U)$, the functions $X_{i}(\alpha)$, $i=1, \ldots, m$, are identically zero on $\pi(U)$. The family $X_{1}, \ldots, X_{m}$ being a Liebracket generating family, we thus obtain that $\alpha$ is locally constant.

2.4. A remark on Lemma 2.3. Let $\hat{\alpha}$ be the real-valued function on $\hat{M}$ defined by

$$
\left\{\begin{array}{l}
\hat{\alpha}(0)=\alpha\left(q_{0}\right), \\
\hat{X}_{i}(\hat{\alpha}) \equiv X_{i}(\alpha)\left(q_{0}\right) \quad i=1, \ldots, m, \\
\hat{X}_{k}(\hat{\alpha}) \equiv 0, \quad k=m+1, \ldots, n .
\end{array}\right.
$$

In a system of privileged coordinates $z$ at $q_{0}$ such that $X_{i}\left(z_{j}\right)\left(q_{0}\right)=\delta_{i j}$, $\hat{\alpha}$ writes as

$$
\hat{\alpha}=\alpha\left(q_{0}\right)+\sum_{i=1}^{m} z_{i} X_{i}(\alpha)\left(q_{0}\right) .
$$

The existence of the mapping $\Psi$ in Lemma 2.3 may be interpreted as follows.

Lemma 2.5. There exists a fiber-preserving map $\hat{\Phi}: T^{*} \hat{M} \rightarrow T^{*} \hat{M}$ such that, on a neighbourhood of every ample covector (w.r.t. $\hat{g}$ ), $\hat{\Phi}$ is smooth and sends the integral curves of the Hamiltonian vector fields of the metric $\hat{g}$ to the ones of $\hat{\alpha}^{2} \hat{g}$.

Proof. Note that $\hat{d}$ is the vector $d$ constructed by using $\left\{\hat{X}_{1}, \ldots, \hat{X}_{n}\right\}$ as a frame and $\hat{\alpha}$ as conformal coefficient in (2.11). Let $\Psi$ be the solution of $\hat{A} \Psi=\hat{d}$ and set

$$
\left\{\begin{array}{l}
\hat{\Phi}_{k}=\hat{\alpha} u_{k}, \quad k=1, \ldots, m, \\
\hat{\Phi}_{k}=\Psi_{k}+\hat{\alpha} u_{k}, \quad k=m+1, \ldots, n .
\end{array}\right.
$$

Define $\hat{\Phi}: T^{*} \hat{M} \rightarrow T^{*} \hat{M}$ as the fiber-preserving map such that $u \circ \hat{\Phi}=\left(\hat{\Phi}_{1}, \ldots, \hat{\Phi}_{n}\right)$. It results from [5, Proposition 3.13] that $\hat{\Phi}$ sends the extremal flows of $\hat{g}$ to the one of $\hat{\alpha}^{2} \hat{g}$ near any ample covector. 


\section{The most General SUb-RIEMANNiAn WeYL TYPE THEOREM}

In this section we formulate the most general technical version of the subRiemannian Weyl theorem that we were able to obtain. The versions of the subRiemannian Weyl theorem (Theorems 1.10 and 1.11) formulated in the Introduction will follow from its proof.

We start with the following.

Definition 3.1. Given an open subset $U$ of $\mathbb{C} M$ the function $\delta$ on the complexified cotangent bundle $T^{*} U$ is called a polynomial with respect to the fibers over $U$ if, in the canonical coordinates induced by some local coordinates in $U, f$ is represented as a polynomial with respect to the fibers with coefficients being holomorphic function of the base $U$. Further, given a point $q_{0} \in M$ a germ over $q_{0}$ of polynomials with respect to the fibers of cotangent bundle is an equivalence class of such polynomials so that two polynomials are equivalent if they coincide over a neighborhood of a point $q_{0}$.

Theorem 3.2. Assume that $(M, D, g)$ is a real analytic sub-Riemannian manifold such that there is no non-constant polynomial $\delta$ with respect to the fibers of $T^{*} U$ over some open set $U$ of $\mathbb{C} M$, such that an open subset of the zero-level set of $\delta$ is a manifold foliated by complex normal extremals each of which projects to non-strictly normal geodesics. Then the sub-Riemannian metric $g$ is Weyl projectively rigid.

Proof. Consider the map $\Psi=\widetilde{\Phi}-\alpha \widetilde{u}$, which is a solution of (2.10). Then by Proposition 2.2, to get the conclusion of our theorem we only need to show that $\Psi$ is polynomial with respect to the fibers near a point $q_{0}$.

Given a positive integer $k$ denote by $A_{k}$ the truncation up to the $k$ th layer of the fundamental matrix $A$ from (2.10). By [5, Proposition 3.11] we can choose $k \geq n-m$ large enough so that at least one minor of size $(n-m) \times(n-m)$ in $A_{k}$ is not identically zero.

From now on we work on the complexified manifold $\mathbb{C} M$. The corresponding complexified cotangent bundle $T^{*} \mathbb{C} M$ can be identified locally with $\mathbb{C}^{n} \times \mathbb{C}^{n}$ (where $q_{0}$ is identified with 0 ). Let $\mathcal{O}_{n}$ be the set of germs of holomorphic functions on $\mathbb{C} M \simeq \mathbb{C}^{n}$ at 0 . Under the above identification, a germ of polynomials with respect to the fibers of $T^{*} \mathbb{C} M$ in the sense of Definition 3.1 can be seen as polynomials on (the second copy of) $\mathbb{C}^{n}$ with coefficients in $\mathcal{O}_{n}$. Since $\mathcal{O}_{n}$ is a factorial ring (or unique factorization domain), the set $\mathcal{O}_{n}\left[z_{1}, \ldots, z_{n}\right]$ of these polynomials form a factorial ring as well (see for instance [6]), which mean that every element can be written as a product of irreducible elements, uniquely up to order and units.

It results from (2.10) that for any nonzero minor $\delta$ of $A_{k}$ we have

$$
\Psi_{i}=\frac{p_{i}}{\delta}, \quad m+1 \leq i \leq n,
$$

where $\delta$ and $p_{i}$ are polynomial in $\mathcal{O}_{n}\left[z_{1}, \ldots, z_{n}\right]$. Canceling the greatest common factor of the collection of polynomials $\left\{\delta, p_{m+1}, \ldots, p_{n}\right\}$, we get a collection of polynomials $\left\{\widetilde{\delta}, \widetilde{p}_{m+1}, \ldots, \widetilde{p}_{n}\right\}$ with the greatest common factor equal to constant and such that

$$
\Psi_{i}=\frac{\widetilde{p}_{i}}{\widetilde{\delta}}
$$

Besides, substituting (3.2) into (2.9) we get

$$
\vec{h}(\widetilde{\delta}) \widetilde{p}_{i} \text { is divisible by } \widetilde{\delta} .
$$

Let us show that under the assumption of Theorem $3.2 \widetilde{\delta}$ is constant. Assuming the converse, there is an irreducible polynomial $\delta_{1}$ in $\mathcal{O}_{n}\left[z_{1}, \ldots, z_{n}\right]$ such that 
$\widetilde{\delta}=\delta_{1}^{s} p$, where $s$ is a positive integer and $p$ is a polynomial such that $p$ and $\delta_{1}$ are coprime. By constructions, there exists $j \in\{m+1, \ldots n\}$ such that $\widetilde{p}_{j}$ is not divisible by $\delta_{1}$, otherwise $\delta_{1}$ is a nonconstant common factor of the collection $\left\{\widetilde{\delta}, \widetilde{p}_{m+1}, \ldots, \widetilde{p}_{n}\right\}$.

Consider this particular $j$. Although the polynomials $\widetilde{p}_{j}$ and $\widetilde{\delta}$ are not coprime in general, if we further reduce the expression (3.2) for $\Psi_{j}$ to the lowest terms (i.e. such that the numerator and denominator will be coprime), then the denominator will be divisible by $\delta_{1}$. Note that in (3.1) we can use any nonzero maximal minor $\delta$ of $A_{k}$ and the expression for $\Psi_{j}$ in the lowest terms is unique and does not depend on the initial choice of the nonzero maximal minor $\delta$. Hence, $\delta_{1}$ is a common divisor of all maximal minors of $A_{k}$.

From (3.3), there holds

$$
\vec{h}(\widetilde{\delta}) \widetilde{p}_{j}=\left(s p \delta_{1}^{s-1} \vec{h}\left(\delta_{1}\right)+\delta_{1}^{s} \vec{h}(p)\right) \widetilde{p}_{j} \text { is divisible by } \widetilde{\delta}=\delta_{1}^{s} p,
$$

which implies that $\left(s p \vec{h}\left(\delta_{1}\right)+\delta_{1} \vec{h}(p)\right) \widetilde{p}_{j}$ is divisible by $\delta_{1} p$, and so that

$$
p \widetilde{p}_{j} \vec{h}\left(\delta_{1}\right) \text { is divisible by } \delta_{1} \text {. }
$$

Since $\widetilde{p}_{j}$ and $p$ are not divisible by the irreducible polynomial $\delta_{1}$, we conclude that $\vec{h}\left(\delta_{1}\right)$ is divisible by $\delta_{1}$.

Denote by $\mathcal{S}_{1}$ the zero-level set of $\delta_{1}$ (it is an analytic subset of $T^{*} \mathbb{C} M \simeq \mathbb{C}^{n} \times \mathbb{C}^{n}$ near the fiber $\left.\{0\} \times \mathbb{C}^{n}\right)$. We have shown that $\vec{h}\left(\delta_{1}\right)$ is zero on $\mathcal{S}_{1}$. Note that, although $\delta_{1}$ is irreducible over $\mathcal{O}_{n}$, its restriction to some fiber might be reducible over $\mathbb{C}$. However, the restriction on the generic fiber (in the domain of definition of $\delta_{1}$ ) is irreducible. This implies that $d \delta_{1}$ is not identically zero on $\mathcal{S}_{1}$. Indeed, assume the converse. Since $\delta_{1}$ is not constant, there exists $k$ such that $\frac{\partial}{\partial u_{k}} \delta_{1}$ is not zero but $\frac{\partial}{\partial u_{k}} \delta_{1}=0$ on $\mathcal{S}_{1}$. By applying the Hilbert Nullstellensatz to each generic fiber $\frac{\partial}{\partial u_{k}} \delta_{1}$ must belong to the radical of the ideal generated by the restriction of $\delta_{1}$ to the same fiber, but this is impossible as degree of the polynomial $\frac{\partial}{\partial u_{j}} \delta_{1}$ is smaller than degree of $\delta_{1}$.

Denote by $\mathcal{S}_{2}$ the subset of $\mathcal{S}_{1}$, where the vector $\left(\frac{\partial \delta_{1}}{\partial u_{1}}, \ldots, \frac{\partial \delta_{1}}{\partial u_{n}}\right)^{4}$ is not equal to zero. By constructions this is an open (and dense) subset of $\mathcal{S}_{1}$. Then, by constructions, $\mathcal{S}_{2}$ is a submanifold of $\mathcal{S}_{1}$ and $\vec{h}$ is tangent to $\mathcal{S}_{2}$. Therefore, any complexified normal extremal of $(M, D, g)$ starting at a point of $\mathcal{S}_{2}$ will stay in $\mathcal{S}_{2}$ for sufficiently small time and $\mathcal{S}_{2}$ is foliated by normal extremals.

Consider such a normal extremal $\lambda(t), t \in[0, T]$, in $\mathcal{S}_{2}$, so that $\delta_{1}(\lambda(t)) \equiv 0$. Given any $\lambda \in T^{*} \mathbb{C} M$ define the filtration $\left\{J_{\lambda}^{(k)}\right\}_{k \in \mathbb{N}}$ as follows. First, set $J_{\lambda}^{(0)}$ to be the tangent space to the fiber of $T^{*} \mathbb{C} M$ at $\lambda$, i.e.

$$
J_{\lambda}^{(0)}=\left\{v \in T_{\lambda}\left(T^{*} M\right): \pi_{*} v=0\right\},
$$

where, as before, $\pi: T^{*} \mathbb{C} M \mapsto \mathbb{M}$ denotes the canonical projection. Note that $J^{(0)}$ defines a distribution on $T^{*} \mathbb{C} M$ called the vertical distribution. Finally, define recursively

$$
J_{\lambda}^{(k)}=J_{\lambda}^{(k-1)}+\operatorname{span}\left\{(\operatorname{ad} \vec{h})^{k} V(\lambda): V \text { is a local section of } J^{(0)}\right\} .
$$

\footnotetext{
${ }^{4}$ Here we could impose a weaker condition $d \delta_{1} \neq 0$ but we will need the given stronger condition in the next section
} 
Since $\delta_{1}$ is a common divisor of all maximal minors of $A_{k}$, due to [5, Lemma 3.12] there holds $\operatorname{dim} J_{\lambda(t)}^{(k+1)}<2 n$ for all $t \in[0, T]$, and so

$$
\operatorname{dim} J_{\lambda(t)}^{(n-m)}<2 n \quad \forall t \in[0, T]
$$

(recall that from the beginning we have taken $k \geq n-m$ ). Further, for $t$ in an open and dense subset of $[0, T]$ the dimensions of the spaces $J_{\lambda(\tau)}^{(n-m)}$ are constant for every $\tau$ sufficiently close to $t$. This and (3.4) imply that for these times $t$ we have, for any $s \in \mathbb{N}, \operatorname{dim} J_{\lambda(t)}^{(s)} \leq \operatorname{dim} J_{\lambda(t)}^{(n-m)}<2 n$. As a consequence, $\left.\gamma(\cdot)\right)=\pi(\lambda(\cdot))$ is a non-strictly normal geodesic of $(M, D, g)$ (it results from [1, Prop. 3.12] since our sub-Riemannian manifold is analytic). This completes the proof of our theorem as we can take $\mathcal{S}_{2}$ as the required open set in the zero-level set of $\delta$ and $U=\pi\left(\mathcal{S}_{2}\right)$.

\section{Proof of TheOrems 1.10 AND 1.11}

First we prove Theorem 1.11. Assume by contradiction that the metric $g$ is not Weyl projectively rigid. Then by Proposition $2.2 \Psi$ is not a polynomial with respect to the fibers and we can repeat the arguments of proof of Theorem 3.2 in the previous section to find a polynomial $\delta_{1}$ such that the open set $\mathcal{S}_{2}$ of the zerolevel set of $\delta_{1}$ is foliated by non-strictly normal complex geodesics. We consider two cases separately.

Case $1 \mathcal{S}_{2}$ does not belong to any level set of $h$. Then there exists a nonzero $c \in \mathbb{C}$ such that $\mathcal{S}_{2}$ is transversal to the $c$-level set of $h$ at some point $\lambda_{0}$. By construction of $\mathcal{S}_{2}$ the vector $\left(\frac{\partial \delta_{1}}{\partial u_{1}}, \ldots, \frac{\partial \delta_{1}}{\partial u_{n}}\right)$ is not zero. Then for every point $q \in M$ sufficiently close to $\pi\left(\lambda_{0}\right)$, the intersection of an open subset of $\mathcal{S}_{2}$ (that for simplicity of notation will be called $\mathcal{S}_{2}$ in the sequel) with the fiber $T_{q}^{*} \mathbb{C} M$ and the $c$-levels set of $h$ is of dimension $n-2$.

It gives rise to a $(n-2)$-parameters family of complexified normal extremals which project to non-strictly normal geodesics passing through $q$. The corank of these geodesics may vary, but there is an integer $\kappa$, an $\varepsilon>0$, and an open subset $V$ of $\mathcal{S}_{2} \cap T_{q} \mathbb{C} M \cap h^{-1}(c)$ such that for every $\lambda \in V$ the extremal $\left\{e^{t \vec{h}} \lambda,|t|<\varepsilon\right\}$ projects to a geodesic of corank $\kappa$. Thus we proved that there exists, for at least one positive integer $\kappa$, an $(n-2-\kappa)$-parametric family of corank $\kappa$ non-strictly normal complexified geodesics through a point, which contradicts our assumption. This concludes the proof of Theorem 1.11 in the considered case.

Case $2 \mathcal{S}_{2}$ belongs to a c-level set of $h$ for some $c \in \mathbb{C}$. Note that $\mathcal{S}_{2}$ and $h^{-1}(c)$ have the same dimension, so $\mathcal{S}_{2}$ is an open subset of $h^{-1}(c)$. Then for every point $q \in \pi\left(\mathcal{S}_{2}\right)$ the intersection of an open subset of $\mathcal{S}_{2}$ (that for simplicity of notation will be called $S_{2}$ in the sequel) with the fiber $T_{q}^{*} \mathbb{C} M$ is of dimension $n-1$. Then we can arrive to a contradiction in the same way as in the last paragraph of the previous case.

Now let us prove Theorem 1.10. Let $(\hat{M}, \hat{D}, \hat{g})$ be the nilpotent approximation of $(M, D, g)$ at $q_{0}$, and $\Psi$ be the map given by Lemma 2.3. If we will show that $\Psi$ is polynomial, then the conclusion of the theorem will then follow from Lemma 2.4 and the same argument as in the end of the proof of Proposition 2.2. To prove that $\Psi$ is polynomial we can literally repeat the arguments of the proof of Theorem 3.2 and of the previous two paragraphs for the nilpotent approximation $(\hat{M}, \hat{D}, \hat{g})$. 


\section{PROOF OF WeYL'S TYPE THEOREMS IN TERMS OF ABNORMAL EXTREMALS}

5.1. Abnormal extremals and their properties. First, describe the complex abnormal extremals of a distribution $D$ of rank $m$. The construction is the complexification of the standard geometric construction of usual (i.e. real) abnormal extremals from Optimal Control Theory (for details, see for example [2] and [4]).

Let $\sigma$ be the 2-form on $T^{*} \mathbb{C} M$ which is the complexification of the standard symplectic structure on $T^{*} M$. Also denote by $(T \mathbb{C} M)^{\perp}$ the zero section of $T^{*} \mathbb{C} M$.

Definition 5.1. An unparametrized Lipschitzian complex curve in $\mathbb{C} D^{\perp} \backslash(T \mathbb{C} M)^{\perp}$ is called an abnormal extremal of a distribution $\mathbb{C} D$ if the (complex) tangent line to it at almost every point belongs to the kernel of the restriction $\left.\sigma\right|_{\mathbb{C} D^{\perp}}$ of $\sigma$ to $\mathbb{C} D^{\perp}$ at this point.

Directly from this definition it follows that any abnormal extremal belongs to the set

$$
\widetilde{W}_{D}:=\left\{\lambda \in \mathbb{C} D^{\perp}: \operatorname{ker}\left(\left.\sigma\right|_{\mathbb{C} D^{\perp}}(\lambda)\right) \neq 0\right\} .
$$

Since codim $D^{\perp}=\operatorname{rank} D=m$, from the elementary properties of skew-symmetric matrices and forms it follows that

$$
\widetilde{W}_{D}= \begin{cases}\mathbb{C} D^{\perp} & \text { if } m \text { is odd, } \\ \left\{\lambda \in \mathbb{C} D^{\perp}:\left(\bigwedge^{n-m / 2}\left(\left.\sigma\right|_{\mathbb{C} D^{\perp}}\right)\right)(\lambda)=0\right\} & \text { if } m \text { is even, }\end{cases}
$$

where $\bigwedge^{s}\left(\left.\sigma\right|_{\mathbb{C} D^{\perp}}\right)$ denotes the $s$ th wedge-power of the form $\left.\sigma\right|_{\mathbb{C} D^{\perp}}$. Note that for every odd rank distributions and for generic even rank distributions

$$
\operatorname{dim} \widetilde{W}_{D}= \begin{cases}2 n-m & \text { if } m \text { is odd, } \\ 2 n-m-1 & \text { if } m \text { is even }\end{cases}
$$

Note that in general for even rank distribution $D$ the set $\widetilde{W}_{D}$ is not smooth at every point but it is smooth for generic distributions of this class.

Now define the following subset $W_{D}$ of $\widetilde{W}_{D}$ as follows:

$$
W_{D}:=\left\{\lambda \in \widetilde{W}_{D}: \operatorname{ker}\left(\left.\sigma\right|_{\widetilde{W}_{D}}(\lambda)\right) \text { is one-dimensional }\right\} .
$$

(for the detailed description of the set $W_{D}$ in terms of a local basis of distribution see $[4])$.

By constructions, the kernels of $\left.\sigma\right|_{W_{D}}$ form the characteristic complex rank 1 distribution $\mathcal{C}$ on $W_{D}$ and the complex integral curves of this distribution are complex abnormal extremals of the distribution $D$. Moreover, these are all complex abnormal extremals which lie entirely in $W_{D}$. Following [2], we give the following definition.

Definition 5.2. We say that a complex abnormal extremal $\Gamma$ and the corresponding abnormal geodesics are of minimal order if the set $\Gamma \cap W_{D}$ has a full measure in $\Gamma$.

Remark 5.3. Since $W_{D}$ is open, the set $\Gamma \cap W_{D}$ is open in $\Gamma$.

5.2. The key lemma making the passage from normal to abnormal extremals. First we prove the following.

Lemma 5.4. Let $D$ be a real analytic distribution such that every complex abnormal extremal of $D$ is of minimal order. Assume that for a sub-Riemannian metric $g$ on $D$ there exists a nonzero polynomial $\delta$ with respect to the fibers of $T^{*} U$ over some open set $U$ of $\mathbb{C} M$, such that an open subset of the zero-level set of $\delta$ is foliated by complex normal extremals of $g$ which are lifts of non-strictly normal geodesics. Then 
there exists a submanifold $\mathcal{S}_{0}$ of codimension one in the projectivization $\mathbb{P} T^{*} \mathbb{C} M$ of $T^{*} \mathbb{C} M$ foliated by complex abnormal extremals of D. Moreover, we can choose $\mathcal{S}_{0}$ such that it belongs to the projectivization $\mathbb{P} W_{D}$ of $W_{D}$.

Proof. Let $\mathcal{S}$ be a smooth part of the zero level set of $\delta$. Given $\lambda \in \mathcal{S}$ take the geodesic $\gamma_{\lambda}$ obtained by the projection to $M$ of the sub-Riemannian extremal passing through $\lambda$ and let $\mathcal{A}_{\lambda}$ be the affine space of all normal lifts of $\gamma_{\lambda}$. Note that $\mathcal{A}_{\lambda}$ can be seen as an affine subspace of $T_{\pi(\lambda)}^{*} \mathbb{C} M$, as any lift is uniquely defined by its intersection with $T_{\pi(\lambda)}^{*} \mathbb{C} M$. Also, clearly $\lambda \in \mathcal{A}(\lambda)$, and by the non-strictly normal assumption, $\operatorname{dim} \mathcal{A}_{\lambda}>0$. This dimension may vary, but there is an integer $\kappa>0$ and an open subset $V$ of $\mathcal{S}$ such that $\operatorname{dim} \mathcal{A}_{\lambda}=\kappa$ for every $\lambda \in V$. Further, let $\mathcal{B}_{\lambda}=\mathcal{S} \cap \mathcal{A}_{\lambda}$. Let $\mathcal{E}_{\lambda}$ be the vector space corresponding to the affine space $\mathcal{A}_{\lambda}$ and $\mathbb{P} \mathcal{E}_{\lambda}$ its projectivization. In the sequel, given an element $e \neq 0$ of a vector space, $[e]$ will denote the line generated by $e$, i.e. the element of the corresponding projective space. Note that the line $[\lambda]$ in $T_{\pi(\lambda)}^{*} \mathbb{M}$ does not belong to $\mathcal{B}_{\lambda}$, because two extremals passing through two different points of the line $[\lambda]$ project to the same unparametrized curve, but the parametrizations on this curve, induced by these two projections, are different (one is a non-unit scalar multiple of the other one).

Let us distinguish the following pair of alternative properties:

(1) $\mathcal{B}_{\lambda}$ is a $(\kappa-1)$-dimensional manifold;

(2) $\mathcal{B}_{\lambda}$ is a $\kappa$-dimensional manifold;

It is easy to see that, maybe after shrinking $V$, either property (1) or property (2) hold simultaneously for every $\lambda \in V$.

Take some codimension 1 submanifold $W$ of $V$. If $\kappa>1$ we take $W$ being transversal to $\mathcal{B}_{\lambda}$ for every $\lambda$. For every $\lambda \in W$ choose $f(\lambda) \in \mathcal{A}_{\lambda}$ smoothly in $\lambda$ and such that the restriction of the map $\lambda \mapsto[f(\lambda)-\lambda]$ to the set $\mathcal{B}_{\lambda} \cap W$ is injective. The latter is possible because

$$
\operatorname{dim}\left(\mathcal{B}_{\lambda} \cap W\right) \leq \operatorname{dim} \mathbb{P} \mathcal{E}_{\lambda} .
$$

Indeed, the right hand-side of (5.2) is equal to $\kappa-1$, whereas the left hand-side of (5.2) is equal to $\kappa-2$ if condition (1) holds with $\kappa>1$; or to $\kappa-1$ if either condition (2) or condition (1) with $\kappa=1$ holds.

By Remark 1.9 there is a projectivized abnormal lift $\Gamma_{\lambda}$ of $\gamma_{\lambda}$ passing through $[f(\lambda)-\lambda]$. Let

$$
\mathcal{S}_{0}=\bigcup_{\lambda \in W} \Gamma_{\lambda}
$$

The imposed injectivity conditions ensures that to different normal extremals, foliating $\mathcal{S}$, different abnormal extremals are assigned. However, in general the map $\lambda \mapsto[f(\lambda)-\lambda]$ on the whole $W$ is not injective so that the family $\left\{\Gamma_{\lambda}\right\}_{\lambda \in W}$ of curves in general do not foliate $\mathcal{S}_{0}$, because some of them intersect. However, now we can use the assumption that all abnormal extremals are of minimal order: fix $\lambda_{0} \in W$, then there exists $\lambda \in \Gamma_{\lambda_{0}} \cap \mathbb{P} W_{D}$, where $\mathbb{P} W_{D}$ is the projectivization of $W_{D}$. By Remark 5.3 there exists a connected neighborhood $V_{0}$ of $\lambda$ in $\mathcal{S}_{0} \cap \mathbb{P} W_{D}$ and this neighborhood is foliated by (connected components) of $\Gamma_{\lambda} \cap V_{0}$. This is the codimension one submanifold of $\mathbb{P} T^{*} \mathbb{C} M$ desired in the lemma.

5.3. Proof of Theorem 1.14. Assume by contradiction that the metric $g$ is not Weyl projectively rigid. Then by Theorem 3.2 there exists a polynomial $\delta$ such that the open level set $\mathcal{S}$ of its zero-level set is foliated by non-strictly normal complex geodesics. Then we can choose a codimension 1 submanifold $\mathcal{S}_{0}$ of $\mathbb{P} T^{*} \mathbb{C} M$ as 
in Lemma 5.4. This means that $\operatorname{dim} \mathcal{S}_{0}=2 n-2$. On the other hand $\mathcal{S}_{0}$ is a submanifold of $\mathbb{P} W_{D}$ and by (5.1) for $m>1$ we have

$$
2 n-2=\operatorname{dim} \mathcal{S}_{0} \leq \operatorname{dim} \mathbb{P} \widetilde{W}_{D} \leq 2 n-3,
$$

which leads to the contradiction.

5.4. Proof of Theorem 1.13. Now let us prove Theorem 1.10. Let $(\hat{M}, \hat{D}, \hat{g})$ be the nilpotent approximation of $(M, D, g)$ at $q_{0}$, and $\Psi$ be the map given by Theorem 3.2. If we will show that $\Psi$ is polynomial, then the conclusion of the theorem will then follow from Lemma 2.4 and the same argument as in the end of the proof of Proposition 2.2. To prove that $\Psi$ is polynomial we can literally repeat the arguments of the proof of Theorem 3.2 and of the previous subsection for the nilpotent approximation $(\hat{M}, \hat{D}, \hat{g})$.

5.5. A refinement of the condition of minimal order. Assume that there exists a nested set collection $\left\{\widetilde{W}_{D}^{(i)}\right\}_{i=0}^{N}$ in $\mathbb{P} D^{\perp}$ such that the following conditions hold:

(1) $\widetilde{W}_{D}^{(0)}=\widetilde{W}_{D}$

(2) $\widetilde{W}_{D}^{(i+1)} \subsetneq \widetilde{W}_{D}^{(i)}$ for every $i \in\{0, \ldots N-1\}$;

(3) The set $W_{D}^{(i)}:=\widetilde{W}_{D}^{(i)} \backslash \widetilde{W}_{D}^{(i+1)}$ is a manifold and $d_{i}:=\operatorname{dim} W_{D}^{(i)}$ is strictly decreasing with respect to $i, i \in\{0, \ldots N-1\}$;

(4) - If $d_{i}$ is odd, then the kernel of restriction of the canonical symplectic form $\sigma$ to $W_{D}^{(i)}$ is one-dimensional at every point of $W_{D}^{(i)}$;

- If $d_{i}$ is even, then the subset $\bar{W}_{D}^{(i)}$ of $W_{D}^{(i)}$, consisting of all points for which the restriction of $\sigma$ to $W_{D}^{(i)}$ has nonzero kernel, is a codimension one submanifold of $W_{D}^{(i)}$ such the kernel of the restriction of $\sigma$ to $\bar{W}_{D}^{(i)}$ is one-dimensional at every point of $\bar{W}_{D}^{(i)}$

Definition 5.5. We say that a complex abnormal extremal $\Gamma$ and the corresponding abnormal geodesics have weakly minimal order if there exist $i \in\{0, \ldots, N\}$ such that the set $\Gamma \cap W_{D}^{(i)}$ has a full measure in $\Gamma$.

Obviously a complex abnormal extremal of minimal order has also weakly minimal order (it just corresponds to $i=0$ in Definition 5.5)

From the proof of Lemma 5.4 it follows immediately that we can replace the condition that all abnormal extremals of $D$ are of minimal order by the condition that all abnormal extremals of $D$ have weakly minimal order in the formulation of Lemma 5.4 and consequently in the formulations of Theorems 1.13 and 1.14 and Corollary 1.15 .

\section{REFERENCES}

[1] A. Agrachev, D. Barilari, L. Rizzi, Curvature: a variational approach, Memoirs of the AMS, 256 (2018), no. 1225.

[2] Y. Chitour, F. Jean, E. Trélat, Genericity results for singular curves, J. Differential Geom. Volume 73, Number 1 (2006), 45-73.

[3] Y. Chitour, F. Jean and E. Trélat, Singular trajectories of control-affine systems, SIAM Journal on Control and Optimization, 47 (2), 2008.

[4] B. Doubrov, I. Zelenko, On local geometry of vector distribution with given Jacobi symbols, preprint, submitted, arXiv:1610.09577 [math.DG], 56 pages.

[5] F. Jean, S. Maslovskaya and I. Zelenko, On projective and affine equivalence of subRiemannian metrics, accepted in Geom. Dedicata, eprint arXiv:1801.04257, 2018.

[6] T. de Jong and G. Pfister, Local analytic geometry: Basic theory and applications, Advanced Lectures in Mathematics, Friedr. Vieweg \& Sohn, Braunschweig, 2000.

[7] T. Levi-Civita, Sulle trasformazioni delle equazioni dinamiche, Ann. Mat. Ser. 2a, 24, 255300,1896 
[8] H. Weyl, Zur Infinitisimalgeometrie: Einordnung der projektiven und der konformen Auffasung, Nachrichten von der K. Gesellschaft der Wissenschaften zu Göttingen, MathematischPhysikalische Klasse, 1921; Selecta Hermann Weyl, Birkäuser Verlag, Basel und Stuttgart, 1956.

[9] I. Zelenko, On the geodesic equivalence of Riemannian metrics and sub-Riemannian metrics on distributions of corank 1, Sovrem. Mat. Prilozh. No. 21, Geom. Zadachi Teor. Upr., 79105, 2004; Engl.transl.: J. Math. Sci. (N. Y.) 135 (4), 3168-3194, 2006.

Frédéric Jean, Uma, EnSta Paris, Institut Polytechnique de Paris, F-91120 Palaiseau, FRANCE

E-mail address: frederic.jean@ensta-paris.fr

$U R L:$ http://uma.ensta-paris.fr/ fjean

Sofya Maslovskaya, inRIa Sophia Antipolis, team Biocore, 2004 Route des Lucioles, BP93 06902 Sophia Antipolis Cedex, France

E-mail address: Sofya.Maslovskaya@inria.fr

Igor Zelenko, Department of Mathematics, Texas A\&M University, College Station, TEXAS 77843, USA

E-mail address: zelenko@math.tamu.edu

$U R L:$ http://www.math.tamu.edu/ zelenko 